\title{
Norois
}

Environnement, aménagement, société

\section{Politiques d'aménagement et élus sur le littoral}

Le camping-caravaning sur parcelles privées : vraie question, faux problème?

Planning and local representatives on the coastal zone Caravaning on private plots: real question, false problem?

\section{Clotilde Buhot et Yann Gérard}

\section{(2) OpenEdition}

Journals

Édition électronique

URL : https://journals.openedition.org/norois/4513

DOI : $10.4000 /$ norois.4513

ISBN : 978-2-7535-2634-1

ISSN : $1760-8546$

Éditeur

Presses universitaires de Rennes

\section{Édition imprimée}

Date de publication : 30 décembre 2012

Pagination : 63-74

ISBN : 978-2-7535-2633-4

ISSN : 0029-182X

\section{Référence électronique}

Clotilde Buhot et Yann Gérard, «Politiques d'aménagement et élus sur le littoral », Norois [En ligne], 225 | 2012, mis en ligne le 30 décembre 2014, consulté le 14 janvier 2022. URL : http:// journals.openedition.org/norois/4513; DOI : https://doi.org/10.4000/norois.4513 


\title{
Politiques d'aménagement et élus sur le littoral Le camping-caravaning sur parcelles privées : vraie question, faux p roblème?
}

\author{
Planning and Local Representatives on the Coastal Zone \\ Caravaning on Private Plots: Real Question, False Problem?
}

\author{
Clotilde Bunot ${ }^{* a}$, Yann GÉrard ${ }^{\text {b }}$
}

\author{
*Auteur correspondant \\ aADEF, 7, avenue de la République - 75011 PARIs (clotilde.buhot@adef.org) \\ b ADEF - revue études foncières, 7, avenue de la République - 75011 Paris(yann.gerard@adef.org)
}

Résumé : Le camping-caravaning sur parcelles privées cultive les paradoxes. À la fois massif et mal connu, il constitue également un point noir des politiques d'aménagement, puisqu'il continue à être l'objet de politiques de résorption qui ne fonctionnent pas. Les travaux sur la question concluent globalement à l'inadéquation entre la réglementation et la réalité du terrain, toujours complexe et nécessitant des arrangements locaux. Afin de mieux comprendre ce point noir dans les politiques d'aménagement, nous proposons une analyse qui ne pose pas le camping-caravaning sur parcelles privées présent dans les communes littorales comme un problème, mais comme une pratique différemment acceptée par les élus locaux. Nous montrons que l'acceptation de cette pratique est très variable, le camping-caravaning sur parcelles privées (CCPP) est même considéré comme peu problématique par les élus. Les questions en lien avec la légalité du CCPP n'interviennent que dans un second temps. Une typologie permet, de plus, de conclure à une forte variabilité de la nature du CCPP sur le littoral français. Cette analyse souligne ainsi la pertinence de la mise en avant des points de vue des élus locaux pour comprendre les difficultés des politiques publiques. Elle rappelle également l'importance de penser l'aménagement de façon horizontale, par opposition à une logique verticale, qui renvoie exclusivement aux relations local/État.

\begin{abstract}
Camping and caravanning on private plots cultivates contradictions. At the same time a massive and little known, it is also a recurring problem for planning policies, as it is yet the object of resorption policies that don't succeed. Researches about this question usually conclude on the hiatus between law and local context, that is always complex and thus requires "arrangements" between local actors. To better understand this situation, our analysis doesn't consider a priori camping-caravanning on private plots as a problem, but as a phenomenon, which perception is various among local authorities. We show that it is the level of acceptance that characterizes the practice; and this, more than the question of the legality of the practice. A typology permits to conclude to a high variability of the nature of the phenomenon in space. This analysis emphasizes the relevance of taking into account the different point of view to understand the difficulties of public policies. It also underlines the necessity of thinking planning in a "horizontal" way, between local municipalities, opposing to vertical way of thinking, that refers to a state/municipality relation.
\end{abstract}

Mots clés : Politiques d'aménagement - élus locaux - camping-caravaning sur parcelles privées - littoral

Keywords: Planning - local authorities - camping-caravanning on private plots - coastal zone 


\section{INTRODUCTION : LE CCPP UNE VRAIE QUESTION D'AMÉNAGEMENT}

Le camping-caravaning sur parcelles privées cultive les paradoxes qui interpellent directement les politiques publiques, notamment en matière d'aménagement ${ }^{1}$.

Le premier d'entre eux est la relative méconnaissance d'une pratique qui concerne plus d'un million de personnes en France (estimation de F. Poulain, 2009). Ce constat est à mettre en regard avec une volonté généralisée, et déjà ancienne, d'éradication du phénomène en raison de son caractère souvent illégal. On touche là à un deuxième paradoxe du CCPP : malgré des efforts permanents (quoique marqués par des phases plus ou moins intenses) de résorption du phénomène (et sa mort annoncée), il continue à poser problème à la puissance publique ${ }^{2}$. Ces problèmes d'ordre urbanistique ont glissé, à partir des années 1970, vers des préoccupations d'ordre environnemental puis, à présent, vers des questions en lien avec la sécurité des personnes ${ }^{3}$. La question sociale (accès aux loisirs littoraux pour les personnes modestes voire logement précaire) n'est pas complètement évacuée, mais certainement minorée face à l'ampleur du phénomène et à la diversité des pratiquants. Le troisième paradoxe que nous souhaitons souligner ici est l'originalité de ce phénomène qui semble se limiter, en Europe Occidentale, à la France. Cet aspect, dans le cadre du littoral français - qui constitue ici notre terrain d'étude - présente un intérêt d'autant plus certain que les côtes françaises sont soumises, parallèlement à une profusion de réglementations (Buhot, Gérard, Brulay et Choblet, 2009), à une loi spécifique : la loi Littoral depuis 1986. Le CCPP donne donc matière à s’interroger sur les politiques d'aménagement et le rôle de ceux qui les portent (notamment l'État).

Les explications généralement avancées pour expliquer la permanence du phénomène, malgré

1. Ce travail s'inscrit dans le cadre d'un programme de recherche Liteau coordonné par l'Adef : ProCoDyf (Propriétés, conflits et dynamiques foncières sur le littoral : la grande propriété et le camping-caravaning sur parcelles privées, 2009-2012).

2. Il suffit, pour s'en convaincre, de suivre la presse quotidienne régionale sur quelques secteurs littoraux, en particulier durant l'été (secteurs de Plougastel-Daoulas - Finistère, de l'̂̂le de Ré - Charente-Maritime, ou encore côte ouest de la Manche).

3. Problèmes d'inondation/submersion, d'érosion, voire d'incendie. Par exemple, la commune de Vias -Hérault - a fait l'objet de nombreux articles de journaux et de commentaires sur la question. les politiques de résorption mises en œuvre ${ }^{4}$, sont multiples : difficultés des élus locaux face à des pratiques parfois anciennes et ancrées, absence d'alternative à ce type de pratique de loisirs relativement bon marché, voire, parfois, pratiques électoralistes favorisant une certaine tolérance.

Elles ont pour point commun de mettre en avant l'importance du contexte local. L'idée sous-jacente est celle de la capacité d'arrangements vis-à-vis de la norme - dictée par l'échelon central via le Code de l'urbanisme - des acteurs locaux. L'examen des pratiques d'aménagement sur le terrain a montré que le cadre normatif ne s'affranchissait jamais complètement des contraintes locales. J.-P. Gaudin (2002) rappelle ainsi les résultats d'études sociologiques réalisées dès les années 1970 et portant précisément sur les rapports entre Services de l'État et municipalités : «Chacun était à la recherche d'adaptations nécessaires de la règle [...]. »

Si nous souscrivons à ce positionnement qui rappelle au passage que l'élu local est le maillon central du pouvoir d'urbanisme ${ }^{5}$, il nous semble que, pour aller plus loin, l'enjeu est d'aborder le CCPP de la façon la plus neutre possible, sans s'enquérir a priori de son adéquation avec le Code de l'urbanisme. Autrement dit, avant de traiter le camping-caravaning sur parcelles privées comme un problème d'urbanisme à résoudre, il nous semble intéressant de le traiter comme une pratique, sociale et spatiale, qui pose question quant à la façon dont elle est perçue et vécue localement. Il s'agit donc ici d'appréhender le regard que portent les politiques publiques et, en particulier, les décideurs locaux (en l'occurrence les maires) sur le CCPP.

Après être revenu sur l'origine de la pratique et son évolution récente, nous présentons les résultats d'une enquête nationale, réalisée auprès des élus communaux du littoral et visant à mieux cerner à la fois la nature du CCPP et la façon dont il est considéré par les élus locaux. Enfin, l'articulation des principaux résultats avec le regard de plusieurs maires interviewés est l'objet de la troisième partie.

\footnotetext{
4. Détaillées plus loin.

5. Délivrance des permis de construire, définition du zonage et utilisation du droit de préemption urbain sont des compétences communales.
} 


\section{UNE PRATIQUE SOCIALE DE MASSE... MÉCONNUE}

\section{À l'origine de la pratique : le camping non réglementé...}

Si la pratique du camping (alors essentiellement sauvage) remonte au début du $\mathrm{xx}^{\mathrm{e}}$ siècle, celui-ci ne devient un phénomène de masse que dans les années 1950. Une décennie plus tard, précise F. Poulain (2009), les terrains de camping existants sont insuffisants pour absorber le nombre de campeurs. Alors que, jusque-là, aucune loi ne réglementait le camping ${ }^{6}$ puisqu'il suffisait d'avoir l'accord du propriétaire pour s'installer sur son terrain, les années 1960 et 1970 voient l'explosion du nombre de pratiquants et coïncident avec les premiers arrêtés préfectoraux encadrant l'installation des campeurs. Ces derniers sont alors essentiellement motivés par la lutte contre l'insalubrité et la garantie de la sécurité publique.

Les campeurs peuvent alors être chassés de terrains dont (et car) ils ne sont pas propriétaires, ce qui décide un certain nombre d'entre eux à acheter une parcelle. F. Poulain attribue à cette époque (milieu des années 1970) l'avènement du campingcaravaning sur parcelles privées.

Au-delà de la réglementation et des formes d'hébergement, la différence avec le camping sauvage, le camping-carisme ou le camping dans un terrain de camping autorisé (municipal ou privé) est l'inscription spatiale de la pratique et, lorsqu'il s'agit de pratiquants propriétaires de parcelles, d'une appropriation forte des lieux. Chaque année, parfois plusieurs fois par an, la parcelle devient alors le lieu de vacances privilégié où se réunissent famille ou amis.

Premier mode d'hébergement pour les vacances des Français avec 1 million de pratiquants, le faible coût d'achat - et d'entretien - par rapport à une résidence secondaire a facilité son développement depuis les années 1970. C'est cet usage dominant et historique qui fonde la définition de départ du camping-caravaning sur parcelles privées ${ }^{7}$, que nous empruntons à France Poulain (2005) : "L'occupation d'une parcelle, par le biais d'une location ou d'une acquisition, par un habitat de

6. Le premier décret date de 1959 et précise que le camping est devenu « d'intérêt général »(Poupard, 1990).

7. La définition recouvre le terme de cabanisation, souvent utilisé sur les rivages méditerranéens. plein air qu'il s'agisse d'une tente, d'une caravane, d'un mobil-home ou d'un camping-car en dehors d'un terrain de camping autorisé. »

\section{...Qui revêt aujourd'hui un caractère protéiforme}

Le terme caractérise des formes très diverses aujourd'hui en France. Par exemple, avec l'essor du camping-carisme (250 000 véhicules immatriculés), nombreux sont les camping-cars à stationner chaque été sur des parcelles de loisirs. Parallèlement, l'hébergement de plein air " traditionnel » (caravane ou tente) semble en perte de vitesse. Les mobilhomes ne sont désormais plus autorisés qu'à l'intérieur des terrains de camping officiels alors qu'on en aperçoit toujours sur des parcelles privées (fig. 1). À cela s'ajoute le fait que le camping-caravaning sur parcelles privées est régulièrement présenté comme un phénomène dual : entre l'habitat de loisir, dont l'occupation est temporaire, et le logement précaire, dont l'utilisation est permanente. Le premier usage serait encore dominant, mais en perte de vitesse par rapport au second. L'utilisation en tant qu'alternative au logement permanent, réservée à des catégories de population qui n'arrivent plus à se loger dans le parc dit classique compte tenu des niveaux de prix $^{8}$ atteints, serait quant à elle marginale.

Toutefois, A. de Goriainoff relevait déjà - en 1985 - qu' « aucune commune ne pourrait envisager leur disparition [de ces formes d'habitat] sans se préoccuper du relogement des habitants. C'est bien une preuve que ce type d'habitat $[\ldots]$ continue à jouer un rôle social et à répondre à une demande » (figure 1).

\section{Un flou renforcé par la difficile application du droit de l'urbanisme}

Un dernier point contribue également à la difficulté d'avoir une vision d'ensemble du phénomène et à complexifier les contours du CCPP : il s'agit d'un relatif laisser-faire vis-à-vis d'une pratique, dont on estime que $80 \%$ des installations sont dans l'illégalité.

Chaque commune a la possibilité de prendre un arrêté d'interdiction, en motivant la nécessité de

8. Aussi bien le niveau des prix à l'achat d'un bien immobilier que celui des loyers pratiqués sur le marché libre. 

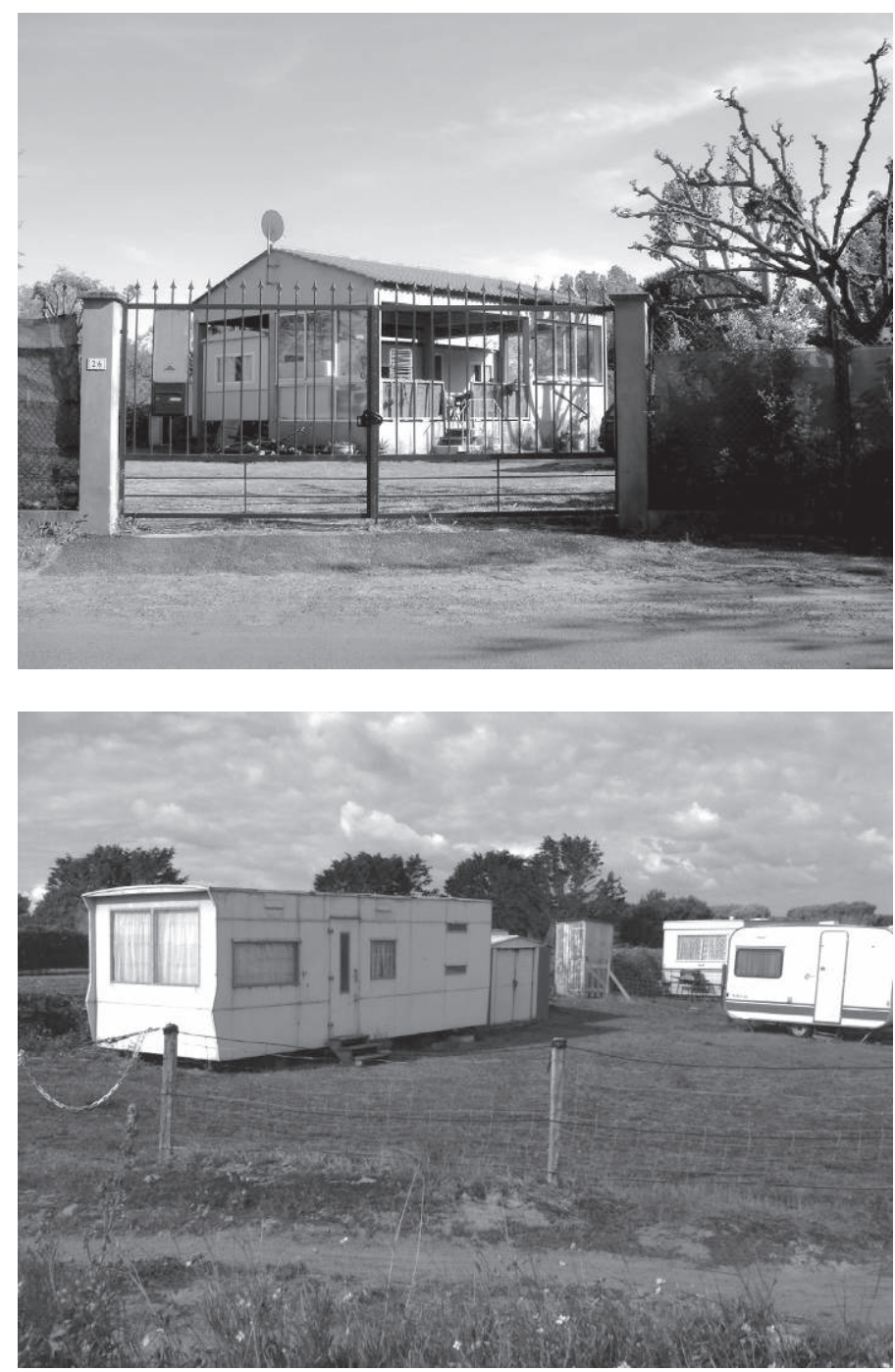

Figure 1 (a et b) : Deux exemples de parcelles accueillant du camping-caravaning - Vias, Hérault. Photo C. Buhot, 2008 (en haut); Réville, Manche. Photo C. Buhot, 2011 (en bas).

Two examples of private plots receiving camping-caravanning

protéger la faune, la flore, l'agriculture ou maintenir la sécurité. En l'absence d'interdiction, « le stationnement est autorisé moins de 3 mois dans l'année sur les parcelles non frappées d'une autre interdiction/disposition (monument historique, bande des 100 mètres...) » (Poulain, 2009); le véhicule devant conserver ses « moyens de mobilité lui permettant d'être déplacé par simple traction » (article R.443-2 du Code de l'urbanisme). Toutefois, dans la pratique, le respect des trois mois de stationnement est, souvent faute de moyens humains, peu contrôlé.

L'attitude des maires, notamment, semble dépendre de facteurs à la fois divers et contingents : volonté politique, relations tissées entre mairie et propriétaires (voire association de propriétaires quand elle existe), attitude de l'administration ${ }^{9}$ à l'égard du CCPP, etc.

De la verbalisation aux poursuites devant le juge pénal, les sanctions à l'encontre des propriétaires sont finalement rares (Poulain, 2003 et Struillou, 2007). Consciente du manque d'efficacité de ces politiques répressives (qui, de plus, génèrent le mécontentement des personnes incriminées), la puissance publique a tenté d'aménager le CCPP en le regroupant, plutôt que de l'éradiquer. De la procédure de Zone d'Aménagement Concertée - à laquelle on a accolé le terme de « à camper »-aux parcs résidentiels de loisirs, en passant par des regroupements en terrains de camping, plusieurs outils ont ainsi vu le jour dans les années 1980 et 1990. Toutefois, peu d'opérations ont été menées à terme (Poulain, 2003). Une des raisons régulièrement évoquée est la volonté d'indépendance et la sensation de liberté offerte par le CCPP, qui se trouverait finalement trop encadré, comme à l'étroit dans les zones aménagées.

Le camping-caravaning sur parcelles privées a ainsi profondément changé de nature en un siècle : à l'origine pratique de loisirs, caractérisée par des abris légers, le CCPP serait aujourd'hui parfois vécu comme une alternative au logement traditionnel. De même autrefois pratique totalement libre, il apparaît clairement encadré par le Code de l'urbanisme. Quelle est la réalité du CCPP? Comment les élus s'accommodent-ils de sa présence sur leur territoire?

\section{La nécessité d'une enquête nationale}

Face à l'absence d'étude globale permettant de cerner le camping-caravaning sur parcelles privées en France ${ }^{10}$, une enquête nationale a été entreprise. Par rapport à des analyses monographiques, qui décrivent parfois avec finesse des jeux d'acteurs, ce travail vise à prendre du recul, en apportant une description du CCPP à l'échelle nationale.

9. Préfecture et DDTM principalement.

10. Nous avons mené un recensement des études réalisées par les DDTM, DREAL, Agences d'urbanisme, Conseils Généraux et Conseils Régionaux, mais les retours ont été décevants car très peu nombreux. Les études auxquelles nous avons eu accès sont très locales : en général à l'échelle d'une intercommunalité. 
L'enquête a été réalisée par le biais d'un questionnaire. Son objectif était double : d'abord proposer des données empiriques de cadrage qui n'existent pas - les études étant en général localisées - mais qui permettent de commencer à aborder la complexité du phénomène. Le questionnaire comprenait à la fois des questions de fait (20 au total) qui visaient à cerner autant que possible une réalité statistique du phénomène, et des questions d'opinion (au nombre de 7) permettant de saisir un début de positionnement des maires vis-à-vis du CCPP. Le questionnaire avait pour second objectif de construire une typologie des communes littorales, en regroupant des modalités de variables permettant de faire ressortir les diverses formes prises par le CCPP.

Cette enquête par questionnaire a ainsi été réalisée auprès de l'ensemble des communes littorales de France métropolitaine (au sens de la définition retenue dans la loi Littoral de 1986), soit près de 1000 collectivités, afin d'obtenir une vue d'ensemble du phénomène. L'envoi du questionnaire ${ }^{11}$ s'est effectué au cours de l'hiver 2010 par voie postale, dans le cadre d'un courrier adressé aux maires. L'échelle nationale a été privilégiée afin d'obtenir un regard suffisamment large; et ce sont les maires qui ont été visés, en raison à la fois de leur connaissance du territoire communal et de leur compétence en matière d'urbanisme. Si les limites de l'enquête par questionnaire sont connues, le taux de retours ainsi que le contenu des réponses ont été satisfaisants. 29,8\% des communes littorales métropolitaines (soit 291 sur 977) ont répondu. La majorité des répondants sont des maires ou des responsables techniques (Directeur général des services ou directeur de l'urbanisme), ce sont donc des personnes informées qui ont rempli le questionnaire. La répartition spatiale des communes ayant répondu est bonne dans la mesure où n'apparaissent pas de vastes secteurs sans réponse. Si certains vides sont remarquables ${ }^{12}$ (la Camargue par exemple), les

11. Le choix du questionnaire s'est imposé pour quatre raisons : l'objet de l'enquête était déjà défini (il s'agissait pour les répondants de décrire la pratique du CCPP sur leur territoire), la facilité et la rapidité à répondre à des questions fermées, la reproductibilité des questions (posées de manière identique à plusieurs centaines d'interlocuteurs), enfin une - relative - facilité de traitement des réponses apportées.

12. D'autant plus que l'absence de réponses de certaines communes peut être interprétée comme une volonté de cacher l'existence du campingcaravaning sur parcelles privées. réponses obtenues permettent néanmoins de mettre en œuvre un traitement des données.

\section{UNE PRATIQUE GLOBALEMENT BIEN ACCEPTÉE}

Au regard des réponses apportées par les 291 communes, le CCPP apparaît encore marqué par un certain dynamisme sur le littoral français : 146 communes ont confirmé la présence de CCPP sur leur territoire (soit la moitié des répondants). Malgré ou en raison - de son aspect protéiforme, il est présent dans toutes les régions françaises (tableau 1), même si le Languedoc-Roussillon arrive en tête (avec $72 \%$ de communes ayant déclaré la présence de CCPP), suivie par la Bretagne $(60 \%)$ et la façade Atlantique $(51 \%)$. Ces proportions confirment le repérage habituel des zones de CCPP relevé par d'autres auteurs (Poulain, 2009).

\begin{tabular}{|c|c|}
\hline Région & $\begin{array}{c}\text { Part des } \\
\text { communes } \\
\text { recensant du } \\
\text { CCPP }(\%)\end{array}$ \\
\hline Nord (Nord-Pas-de-Calais et Picardie) & 23 \\
\hline Normandie (Haute et Basse-Normandie) & 43 \\
\hline Bretagne & 60 \\
\hline $\begin{array}{c}\text { Atlantique (Pays-de-la-Loire, Poitou-Cha- } \\
\text { rentes et Aquitaine) }\end{array}$ & 51 \\
\hline Languedoc-Roussillon & 73 \\
\hline Provence-Alpes-Côte d'Azur & 38 \\
\hline Corse & 32 \\
\hline
\end{tabular}

Tableau 1 : Part des communes recensant du CCPP Lecture : $60 \%$ des communes bretonnes ayant répondu au questionnaire signalent la présence de CCPP sur leur territoire. Source : Questionnaire communes littorales, adef, 2011.

Proportion of municipalities declaring CCPP

En complément des tris à plat, une analyse factorielle des réponses apportées a été réalisée ${ }^{13}$. Elle permet d'isoler les oppositions structurelles caractérisant les dynamiques du CCPP. Les résultats présentés ici portent essentiellement sur les 20 questions de fait ${ }^{14}$.

13. 11 variables actives ont été retenues et 3 axes sont présentés.

14. Dans le questionnaire étaient également présentes 7 questions d'opinions. Nous revenons dans la suite de l'article sur leur exploitation. 


\section{Une pratique relativement discrète?}

Avant la réalisation du questionnaire, nous envisagions deux hypothèses articulées sur deux oppositions :

- un CCPP en augmentation, opposé à un CCPP en régression;

- un CCPP saisonnier, opposé au permanent.

Ces questions apparaissaient en effet primordiales, car elles entretiennent un lien direct avec la gestion publique (dans le cadre du Code de l'urbanisme) du CCPP. Ainsi, la disparition de la pratique (donc sa régression) constitue un objectif de politique d'aménagement. Ensuite, c'est bien le caractère permanent de l'occupation (plus de 3 mois) qui implique, dans de nombreux cas, son incompatibilité avec les textes de loi. Or ces questions liées à ses temporalités (qu'il s'agisse de l'évolution du phénomène ou de sa saisonnalité) n'apparaissent pas primordiales pour décrire les dynamiques du CCPP.

Du questionnaire ressort en premier lieu une profonde opposition ${ }^{15}$ liée à la visibilité du phénomène (fig. 2), puisqu'on retrouve, d'un côté, un CCPP que nous qualifions de discret et, de l'autre, un CCPP que nous qualifions d'ostensible. Or ce dernier est minoritaire. Ainsi, et c'est une surprise, le campingcaravaning sur parcelles privées est plutôt considéré comme discret par les élus, voire ignoré. D’ailleurs, à l'échelle nationale, seule une minorité de communes déclarent prendre en compte le CCPP dans leurs stratégies d'aménagement (seulement $36 \%$ ).

Le CCPP discret, décrit par la majorité des communes, concerne un petit nombre de parcelles sur une petite fraction du territoire communal (moins de 20 parcelles réparties sur moins de 1 ha), il est bien ancré historiquement (la pratique a plus de 20 ans dans plus de $70 \%$ des communes). Les parcelles illégales sont souvent décrites comme peu nombreuses et, de fait, peu de procès-verbaux y sont dressés.

Inversement, l'ostensible concerne environ $20 \%$ des communes. Il est à la fois massif car il concerne plus de 100 parcelles (une trentaine de communes sont concernées) et qu'elles représentent plus de 5 ha (dans un quart des communes); et illégal car les communes déclarent que les parcelles illégales sont «très nombreuses » (22\% des communes) et que des procès-verbaux sont dressés chaque année (17

15. Premier axe factoriel : $\%$ d'inertie $\lambda=16,9 \%$ de l'inertie. d'entre elles signalent en instruire plus de 5 par an). C'est principalement dans ces communes que des arrêtés d'interdiction ont été pris. À ces deux caractéristiques s'ajoute la présence d'associations (signalées dans un quart des communes et généralement sous le régime de la loi 1901). Nous pouvons ici émettre l'hypothèse que leur existence est directement liée à la nécessité des campeurs de se structurer pour s'opposer aux autorités locales. Enfin, et sans surprise, ces modalités sont très liées à une opinion négative des élus en matière d'urbanisme. En effet, les élus interrogés jugent le CCPP négativement («impact négatif à très négatif » dans $90 \%$ des cas) sur les plans paysager, urbanistique, environnemental, sécuritaire et fiscal. D'ailleurs, ils sont $75 \%$ à juger positivement l'intervention publique destinée à gérer le phénomène - qu'elle émane du Conseil Général ou du Conservatoire du littoral.

\section{Les temporalités, secondaires mais au cour des conflits}

Les deux oppositions suivantes (fig. 2 et 3) renvoient à nos hypothèses de départ. Elles mettent en avant à la fois la dynamique et la saisonnalité du CCPP.

Le deuxième axe factoriel ${ }^{16}$ oppose ainsi le CCPP déclinant à l'émergent. Ce résultat contredit les études locales, en faisant apparaître un CCPP émergent bien présent. En effet ces dernières - qu'elles émanent de bureaux d'étude comme BRL (2004) ou AMEDIS (2006), d'agence d'urbanisme (Adeupa, 2006) ou de services de l'État (Préfecture de la Manche, 2007), ont tendance à décrire une stabilisation voire une disparition du phénomène sur le littoral français.

Le déclinant concerne un bon quart des communes, tandis que l'émergent («moins de 10 ans »), associé à une augmentation des parcelles campées et à l'habitat permanent, caractérise $1 / 5^{\mathrm{e}}$ des communes.

Cette composante " habitat permanent " au sein de l'émergent est remarquable car elle pose la question de la nature du CCPP.

Surtout, le troisième axe factoriel ${ }^{17}$, qui oppose le CCPP combattu à l'accepté, montre que l'habitat permanent est lié à la présence de procès-verbaux

16. Deuxième axe factoriel : $\%$ d'inertie $\boldsymbol{\lambda}=9 \%$ de l'inertie.

17. Troisième axe factoriel : $\%$ d'inertie $\lambda=9 \%$ de l'inertie. 

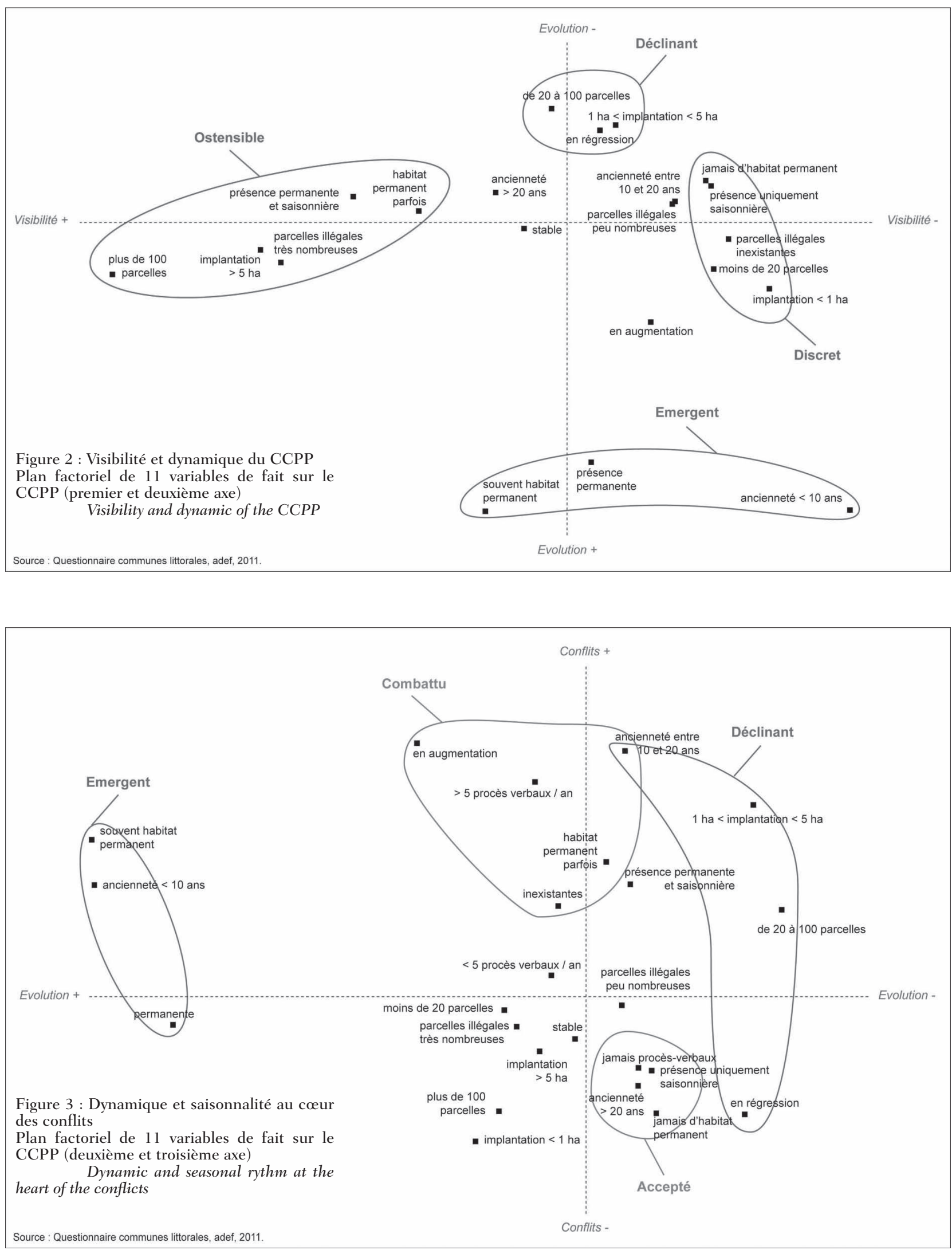
(modalité «plus de 5 procès-verbaux par an »), ce qui n'est pas le cas lorsqu'il s'agit d'une pratique uniquement saisonnière (fig. 3).

Pour conclure, si la forte visibilité du CCPP (ostensible) peut être source de conflits, ce sont surtout la permanence de l'habitat associée à sa progression qui sont corrélées à l'existence de procèsverbaux. Or l'habitat permanent reste encore très minoritaire.

Le CCPP discret reste quant à lui majoritaire et ne semble pas constituer un problème pour les élus.

Cette relative tolérance sur le plan réglementaire ${ }^{18}$ interroge et peut également être interprétée comme résultant d'un manque de moyens pour lutter contre le CCPP illégal; les communes littorales étant souvent de petite taille.

Si les exemples de conflits liés au camping-caravaning sur parcelles privées sont médiatisés, donnant même l'impression que sa pratique est combattue sur tout le littoral français, seule une minorité de communes se déclarent ouvertement en conflit avec les campeurs/propriétaires. La réalisation d'une typologie des communes en fonction de la nature du CCPP dominant permet d'ailleurs de souligner l'hétérogénéité des situations (cf. infra). Elle ouvre ainsi la voie à une réflexion moins manichéenne sur la question et permet de comprendre que, d'une commune à l'autre, l'attitude des élus soit variable.

\section{FACE À L'Hétérogénéité du CCPP, QUELLE COHÉRENCE DES POLITIQUES PUBLIQUES?}

Comment ces grandes oppositions se traduisentelles dans l'espace? La réalisation d'une typologie doit permettre de traduire cartographiquement l'analyse factorielle réalisée. Par ailleurs, des entretiens, réalisés auprès des élus de plusieurs communes, visent à expliquer les différences de positionnement, en fonction des contextes locaux.

\section{Intégré ou conflictuel : une répartition aléatoire du CCPP}

La classification automatique, via une «Classification Ascendante Hiérarchique » $(\mathrm{CAH})$, a été retenue en raison de la simplicité de sa mise

18. Les opinions négatives reposent en majorité sur la qualité esthétique des occupations de parcelles. en œuvre. Elle se fonde sur les résultats de l'analyse factorielle précédente ${ }^{19}$. L'analyse factorielle permet de repérer les variables et modalités structurantes, la CAH permet, ensuite, de les regrouper en classes dans lesquelles se répartissent les communes.

Les deux classes les plus importantes (puisqu'elles regroupent $70 \%$ des communes) correspondent bien à des communes marquées par un CCPP discret et accepté, et donc majoritaire : ce sont les communes tolérantes. Ce qui ne l'empêche pas d'être en régression dans beaucoup d'entre elles. Ces deux classes de communes sont caractérisées par :

- un CCPP «bien implanté stable». Dans ces communes, le CCPP pratiqué paraît à la fois relativement discret et bien présent : sans être exceptionnelle, son ampleur est effective ( 1 à 5 ha, plus de 10 parcelles); il n'est pas structuré en associations et se pratique parfois tout au long de l'année. Cette première classe comprend par exemple GrandcampMaisy $(14)^{20}$, Saint-Méloir-des-Ondes (35) Torreilles (66) ou Aléria (20).

- un CCPP « déclinant ». Cette classe, la plus nombreuse, rassemble 50 communes. C'est à cette dernière que correspond le mieux l'image du CCPP traditionnel : destiné uniquement à de l'habitat temporaire ( $72 \%$ de la classe dans la modalité), utilisé de manière saisonnière et présent depuis longtemps. Les communes concernées dressent rarement des procès-verbaux et les parcelles illégales sont considérées comme peu nombreuses. Enfin, le phénomène est souvent en régression. Dans cette classe, on retrouve des communes comme Montfarville (50), Le Bono (56), Bretignolles sur Mer (85) ou Leucate (11).

On retrouve ensuite les deux classes de communes où le CCPP est potentiellement vecteur de conflits, et donc plus «visible» : ce sont les communes combattives. Elles sont marquées soit par :

- un CPPP « massif stable », d'abord, car d'ampleur importante (nombre de parcelles et superficie), et caractérisé par des parcelles illégales très nombreuses et des associations fréquentes. Parmi les communes représentatives de cette classe figurent Saint-Germain-sur-Ay (50), Plougastel (29), Le Bois Plage en Ré (17) ou encore Vias (34).

19. Les 15 premiers facteurs ont été retenus pour réaliser l'analyse, ces derniers regroupant $90 \%$ de l'information.

20. Les numéros entre parenthèses sont ceux des départements où sont situées les communes citées. 
- enfin, un CCPP « émergent». Avec 14 communes seulement, cette classe est la plus originale. Elle témoigne d'une évolution de la pratique qui remet partiellement en cause la définition initiale proposée. Le CCPP est encore faible dans les communes concernées; toutefois, il est récent (100\% de la classe se trouve dans la modalité «moins de 10 ans ») et en augmentation. Autre caractéristique supplémentaire par rapport aux trois autres classes : il s'agit surtout d'habitat permanent. Les communes de la classe 4 peuvent être représentées par Tardinghen (62), Pont-Aven (56), Elne (66), Six Fours les Plages (83) ou Sari Solenzara (20).

L'ensemble des quatre classes sont représentées sur toutes les façades littorales (tableau 2) : le CCPP apparaît donc partout dans sa diversité. Des oppositions régionales ou nord/sud n'apparaissent pas aussi marquées que prévues. Certaines tendances peuvent être identifiées malgré tout. Les communes combattives sont partout minoritaires, sauf sur la façade Nord/Normandie où elles font jeu égal avec les communes tolérantes. Ce constat est peut-être corrélé à la surreprésentation, inattendue, de la forme émergente du CCPP du MontSaint-Michel à la frontière belge. Il y a là, manifestement, matière à investigation : doit-on y voir le résultat d'un contrôle moins fort de la part de la puissance publique? Que signifie cette demande de logements permanents dans des secteurs où la pénurie de logements est loin d'atteindre celle de la Méditerranée (où le CCPP émergent est également surreprésenté)?

À l'inverse, c'est en Bretagne et sur la façade atlantique que le CCPP régresse le plus. C'est d'ailleurs à l'Ouest qu'il est quasi-exclusivement utilisé pour du loisir (à $90 \%$ contre seulement $10 \%$ en Méditerranée) et c'est également là que les associations sont les plus nombreuses $(75 \%$ localisées du Cotentin à l'île d'Oléron). On retrouve ici l'hypothèse (évoquée supra) d'un phénomène de longue date combattu, et suffisamment menacé pour que des associations se structurent pour tenter de le maintenir.

Parallèlement à ces hypothèses générales, un zoom sur une portion de littoral permet de rappeler, une fois de plus, l'hétérogénéité de la pratique à une grande échelle (figure 4 - planche V). Loin de s'agréger sur plusieurs communes contiguës, les formes de CCPP sont très variables d'une commune à l'autre.

\section{Entre les propriétaires et l'État}

D’un point de vue méthodologique, ce découpage en quatre classes permet d'orienter le choix des sites-test. Ces derniers ont en effet fait l'objet d'enquêtes fines, courant 2011, afin de prolonger la compréhension du phénomène par une démarche davantage dynamique que l'analyse factorielle - qui fournit une photographie statique du phénomène.

Trois sites-test correspondant aux trois premières classes ont été enquêtés ${ }^{21}$. Il s'agit de : l'île de Ré (Charente-Maritime) représentatif du «massif stable », la Côte d'Améthyste (Aude et nord des Pyrénées Orientales) illustrant le «bien implanté stable » et enfin le Val de Saire (Manche) caractéristique du « déclinant». Le contenu des entretiens menés auprès de 9 maires ${ }^{22}$ permet d'af-

\begin{tabular}{|c|c|c|c|c|}
\hline \multirow{2}{*}{} & \multicolumn{2}{|c|}{ Communes tolérantes } & \multicolumn{2}{c|}{ Communes combattives } \\
\cline { 2 - 5 } & \multicolumn{3}{|c|}{ Communes caractérisées par un CCPP } \\
\cline { 2 - 5 } & Bien implanté stable & Déclinant & Massif stable & 24 \\
\hline Nord-Normandie & 24 & 19 & 33 & 8 \\
\hline Bretagne & 21 & 26 & 45 & 4 \\
\hline Atlantique & 17 & 17 & 62 & 16 \\
\hline Méditerranée & 21 & 39 & 24 & \\
\hline
\end{tabular}

Tableau 2 : Répartition des 4 classes de la typologie (en \%) Source : Questionnaire communes littorales, adef, 2011. Sharing of the 4 groups of the typology (in \%)
21. Nous partons en effet de l'hypothèse que le CCPP émergent doit être repérable au sein de ces derniers.

22. Il s'agit des maires des communes du Bois-Plage, La Couarde, La Flotte et Rivedoux sur l'île de Ré, de Torreilles et Elne pour la Côte d'Améthyste et de Gatteville, Montfarville et Réville dans le Val de Saire. Une série d'autres entretiens visant les propriétaires a également été réalisée, elle n'est pas développée ici. 
finer la position des élus sur la pratique du CCPP et éclaire sur les raisons de son acceptation, malgré son caractère souvent illégal d'un point de vue juridique.

En définitive, qu'est-ce qui, dans les discours recueillis, justifie le peu d'action et de lutte contre le CCPP? Les élus déploient trois registres d'argumentation : celui de l'impuissance, celui de la proximité domestique avec les campeurs et, enfin, celui de la justice sociale.

Aborder la question du CCPP conduit les élus à exprimer un sentiment d'impuissance. Ce dernier résulte de l'incompréhension des maires face au hiatus existant entre la loi, telle qu'elle est écrite, et son application, et notamment les décisions prises par les autres acteurs susceptibles d'intervenir.

Si les élus locaux se sentent parfois dans une situation délicate, entre d'un côté les campeurs/ propriétaires et de l'autre des acteurs relevant de la sphère publique, c'est en premier lieu parce qu'ils s'interrogent sur le flou entourant le partage des responsabilités dans la gestion du CCPP. Les opérateurs fonciers publics dont l'objectif est la protection des espaces naturels et agricoles existent bien : Safer, Conservatoire du littoral, Conseils Généraux via leur politique d'espaces naturels sensibles. Toutefois, ces derniers ont leurs propres critères et contraintes d'intervention qui ne leur permettent pas toujours d'intervenir massivement sur des secteurs qui sont jugés non prioritaires. D'où des relations souvent ambigües voire tendues entre les maires et les autres acteurs sur le partage des responsabilités. Si dans certaines communes, elles peuvent être satisfaisantes (cas de Gatteville), dans d'autres - plus nombreuses -, les maires se sentent seuls : "personne ne vient nous aider » (maire de Réville).

Toutefois, la situation est encore plus critique quand maires d'un côté et services de l'État et Préfet de l'autre donnent des directives contradictoires voire s'opposent directement. Ces situations antagonistes ont été vécues par certains maires rencontrés. C'est le cas à Gatteville où la maire, l'année suivant son élection, en 1995, s'est trouvée opposée au sous-Préfet. Ce dernier a fait intervenir la gendarmerie sur la commune alors que l'élue avait opté pour une stratégie plus souple de non délivrance de nouvelles autorisations de camper tout en encourageant les campeurs présents à s'en aller.
C'est finalement un sentiment de décalage entre les objectifs et décisions des services de l'État, et ceux des élus locaux, qui ressort des entretiens. La délivrance d'un permis de construire précaire (la construction est destinée à être démontée à court terme), par le préfet de la Manche, dans une zone naturelle proche de secteurs de CCPP inquiète ainsi le maire de Montfarville. L'argument culturel (il s'agit d'une œuvre d'art) n'est pas de nature à vraiment aider ce dernier, qui ne pourra plus faire valoir la stricte inconstructibilité du secteur face aux campeurs.

Comme nous l'indiquions supra, notre enquête nationale révèle que les élus sont loin d'être systématiquement en lutte contre le CCPP. À l'opposé du combat, c'est un argumentaire de proximité domestique qui est avancé pour justifier de la présence du CCPP. Les maires rencontrés parlent notamment de manière positive de la présence des haies qui cachent les caravanes, de leur entretien régulier : "sinon, ces parcelles seraient entièrement délaissées, en friche » (maire de Montfarville). D'autres mettent en avant un certain respect des campeurs face aux lois ainsi que leur rôle économique : "ça fait marcher l'économie locale» (maire du Bois-Plage).

D'ailleurs, même lorsqu'il est combattu, il n'est pas tant condamné pour ce qu'il est que pour ce qu'il implique comme responsabilité de l'élu, et en particulier le devoir de protection de ses concitoyens. C'est particulièrement le cas sur la question des risques : par exemple les inondations dans le Sud de la France (entretien avec le maire de Toreilles).

Par ailleurs, les relations tissées avec les propriétaires et les associations qui les représentent sont parfois cordiales. Dans les 9 communes enquêtées, les propriétaires de parcelles sont le plus souvent des locaux, c'est-à-dire des personnes qui habitent la commune ou les communes limitrophes ou en sont originaires. Les élus mettent souvent en avant ce caractère local pour justifier d'une certaine proximité avec les campeurs.

Il faut enfin souligner les stratégies d'intégration de certains campeurs, via l'impôt. Structurés ou non en associations, certains groupes insistent en effet pour payer les taxes liées notamment à l'enlèvement des ordures ménagères. Ce geste civique peut être une source supplémentaire de justification de la tolérance envers le CCPP. 
Enfin, les élus éprouvent un réel malaise lorsqu’il s'agit de dénoncer certaines installations à l'administration, au regard des conditions sociales des campeurs/propriétaires. Un argumentaire portant sur la justice sociale apparaît alors et devient d'autant plus fort lorsque les élus sont confrontés, sur leur commune, au manque de logements.

De son côté, la justice considère souvent comme secondaires ces délits comparativement à l'ensemble des infractions qu'elle doit juger. En effet, lorsqu'un permis de construire (ou un document d'urbanisme tel le PLU) est attaqué, l'affaire relève du Tribunal Administratif car c'est le document/la décision administrative qui est attaqué. Dans le cas d'un procès-verbal dressé à l'encontre d'une construction illégale ou d'un stationnement non autorisé, c'est le Tribunal Correctionnel qui est compétent. Ce sont alors les personnes qui sont directement mises en cause et poursuivies par le juge pénal... comme le sont les auteurs de délits bien plus graves : violences physiques, cambriolages, etc.

Au-delà de sa justification, cette pratique pose la question de la gestion d'un phénomène spontané, qui échappe aux réglementations habituelles et constitue parfois une réponse aux problèmes de logement. En la matière, les entretiens font clairement ressortir l'absence de gestion intercommunale. Ainsi, parmi les interlocuteurs cités par les maires, reviennent invariablement le Conseil Général, le Conservatoire du littoral, les associations, le Procureur et/ou le Juge, le Préfet et/ou la DDTM. En revanche, les maires des communes limitrophes sont peu évoqués. Leur gestion du CCPP fait l'objet de peu de commentaires. Seules les communes rétaises parlent facilement de leurs voisines, des positions des maires face au CCPP, de leur action et... de leur bilan. En fait, sur l'île de Ré, la lutte contre le CCPP est devenue une antienne (probablement en lien avec la forte médiatisation de l'île en elle-même). Ce qui n'empêche pas les communes du canton sud d'être profondément divisées sur la gestion du CCPP. Les communes hostiles, voire en conflit ouvert avec le CCPP (La Flotte ou SainteMarie) s'opposent à celles qui continuent à préconiser le dialogue (La Couarde, Rivedoux ou Loix).

Si la tolérance des élus est, a priori, très variable d'un territoire à l'autre, la réglementation l'est également au sein d'une même intercommunalité : le stationnement de moins de trois mois est accepté ici mais formellement interdit là. Cette situation explique certainement que la mise au point de politiques intercommunales pertinentes et cohérentes soit difficile.

\section{Conclusion}

L'analyse proposée montre que, globalement, une problématique d'aménagement n'est pas uniquement liée à la réglementation et au respect du droit mais à l'acceptabilité sociale de la pratique en question. Dans le cas du camping-caravaning sur parcelles privées, les questions de temporalité (son évolution dans le temps ainsi que sa saisonnalité), omniprésentes dans les discours en lien avec la légalité de la pratique (question de la lutte contre et de la disparition d'une pratique contrevenant à la législation, ou caractère permanent de l'occupation de la parcelle), n'apparaissent ainsi qu'au deuxième plan pour la description du CCPP.

Le fait marquant et structurant est lié à l'acceptation du phénomène. La typologie produite permet ainsi de mettre en évidence un premier groupe majoritaire (70\% des communes), où le CCPP est discret. Ce CCPP bénéficie par ailleurs d'une attitude plutôt compréhensive de la part des élus, pour lesquels cette pratique fait parfois partie intégrante de la dynamique communale. Le caractère présumé social du CCPP constitue également une source non négligeable d'acceptation. Entre stabilité et déclin relatif, ce mode de CCPP pourrait paraître anodin, sans véritable enjeu. Toutefois, dans les secteurs où il est en déclin, il pose directement la question de la disparition d'un mode d'appropriation populaire du littoral. Il y a là certainement matière à investigations et approfondissements, notamment auprès des campeurs dont les caractéristiques socioprofessionnelles (s'agit-il toujours de populations aux revenus modestes?), les motivations et les stratégies en matière de loisir sont à préciser (volonté de pérenniser ce type de pratique, rôle des héritiers, nouveaux occupants, etc.). Les propriétaires, souvent placés au cœur du système des acteurs locaux de l'aménagement, aux côtés des opérateurs et des élus notamment (Vilmin, 1999), méritent, dans cette perspective, une attention forte.

Ailleurs, lorsque le CCPP est stable, c'est sa pérennité qui peut être interrogée. La pression sur les littoraux se renforçant, la mise en conformité 
des documents d'urbanisme, notamment avec la loi Littoral, continue. Elle fragilise directement la place d'installations hors normes, d'autant que dans certains secteurs du littoral, le CCPP est vecteur de conflits. En effet, le second groupe de communes mis en évidence à travers le questionnaire révèle un CCPP plus problématique en raison de son caractère soit massif soit permanent. Toutefois, bien que très médiatisé, ce dernier reste minoritaire (avec moins d'un tiers des communes). Il faut dire que pour ceux qui choisissent de lutter contre le CCPP, rien n'est simple. Les élus se trouvent ici pris dans un jeu complexe de responsabilités, impliquant des acteurs de nature différente (services de l'État, collectivités territoriales ou encore Justice). Le fait que les contentieux en matière d'occupation illégale soient traités " au pénal », en même temps que des délits plus graves explique, pour une grande part, l'abandon des poursuites par le juge.

Mais de cette lutte difficile contre le CCPP ressort aussi un paradoxe : celui d'une perception et de politiques localement très diversifiées. D'une commune à l'autre, le CCPP est décrit (et donc accepté) de façon très variable ce qui explique qu'il fasse l'objet de politiques qui peuvent être en opposition totale, dans des communes pourtant voisines. Ce constat conduit à souligner les lacunes inhérentes à la grille de lecture classique «central versus local». Si les politiques d'aménagement impliquent des relations verticales, elles posent également des questions d'intégration horizontale. Autrement dit, c'est autant le problème de la cohérence des politiques intercommunales, qui doivent composer avec l'hétérogénéité du local, qui est posée, que celle de la relation communes/État. Au passage, ce constat permet de dépasser le paradoxe de services de l'État qu'on annonce dans le même temps sur le déclin (le désengagement de l'État) et tout-puissants (dès lors qu'il s'agit de faire appliquer la loi Littoral par exemple).

\section{Bibliographie}

Agence de developpement et d'urbanisme du Pays de Brest (ADEUPA), 2006. La maîtrise du caravanage dans les communes littorales du fond de la rade de Brest, vol. 1 Diagnostic (37 p.) et vol. 2 Solutions de substitution et réglementation (26 p.).

Brl (bureau d'Étude), 2004. Connaissance et identification de la cabanisation sur le littoral du Languedoc-Roussillon, Mission « Littoral », 28 p.

Buhot, C., Gérard, Y., Brulay, F., Choblet, C., 2009. Tensions foncières sur le littoral, Rennes, PUR, $174 \mathrm{p}$.

De Goriainoff A., 1985. «La cabanisation : une vieille histoire », Études foncières, n 28, p. 31-34.

Gaudin J.-P., 2002. Pourquoi la gouvernance?, Paris, Presses de Sciences Po, 134 p.

Miossec, A., 1999. « La France a-t-elle construit une politique de gestion intégrée de son littoral? », in Bulletin de l'association des géographes français, $\mathrm{n}^{\circ} 2$, p. 212-220.

Observatoire national de LA PAUVRETÉ ET DE L'EXCLusion SOCiale, 2005. Systèmes locaux de pauvreté - Focus sur la question de la cabanisation, Paris, Amedis/ONPES/SLP, $45 \mathrm{p}$.

Poulain F., 2009. Le camping aujourd'hui en France entre loisir et précarité, Editions de la DDEA de l'Oise, 172 p.

Poulain F., 2005. Le guide du camping-caravaning sur parcelles privées, Paris, Editions Cheminements, 127 p.

Poulain F., 2003. Les territoires du camping, la pré-urbanisation illégale du littoral par l'habitat de loisirs, Thèse de troisième cycle, université de Paris 8, Institut Français d'Urbanisme, $543 \mathrm{p}$.

Poupard A., 1990. "Caravaning et aménagement », in études foncières, $n^{\circ} 46$, p. 19-23.

Préfecture de la Manche, 2007. Le Camping-caravaning illégal : quelle gestion promouvoir à l'échelle de la Côte des Havres?, $9 \mathrm{p}$.

Struillou J.-F., 2004. « L'application du droit pénal de l'urbanisme », dans Les Cahiers du GRIDAUH, n 1 1, p. 145-187.

VILmin T., 1999. L'aménagement urbain en France : une approche systémique, Lyon, Certu, 250 p. 